\title{
GESTIÓN FISCAL Y MONETARIA PARA LA MITIGACIÓN DE LOS IMPACTOS NOCIVOS DE LA PANDEMIA GLOBAL EN PERÚ
}

\author{
FISCAL AND MONETARY MANAGEMENT FOR MITIGATING THE HARMFUL IMPACTS \\ OF THE GLOBAL PANDEMIC IN PERU
}

Nicko Alberto Gomero Gonzales Universidad Nacional Mayor de San Marcos Lima, Perú

ORCID: https://orcid.org/0000-0002-5642-8298 Autor para correspondencia: ngomerog@unmsm.edu.pe

Víctor Ricardo Masuda Toyofuku Universidad Nacional Mayor de San Marcos Lima, Perú ORCID: https://orcid.org/0000-0001-6767-9466 Correo electrónico: vmasudat@unmsm.edu.pe

Luis Arnaldo Saldaña Pacheco Pontificia Universidad Católica del Perú Lima, Perú

ORCID: https://orcid.org/0000-0002-9381-3566

Correo electrónico: saldana.la@pucp.edu.pe

[Recibido: 11/06/2021 Aceptado: 01/12/2021 Publicado: 29/12/2021]

\section{RESUMEN}

Objetivo: Determinar cómo las medidas monetarias y fiscales implementadas bajo escenario de crisis sanitaria global mitigaron los efectos negativos en la economía nacional. Método: Investigación de tipo descriptiva, correlacional, transversal y no experimental. Se utilizó información secundaria obtenida de fuentes oficiales gubernamentales para realizar las mediciones estadísticas. Resultados: Se halló una correlación de Pearson negativa entre la emisión de dinero y la tasa de interés $r=-0.46$, que explica la caída de las tasas de interés ante aumentos del circulante. El mismo tipo de relación se encontró entre la tasa de interés y los créditos empresariales, siendo el coeficiente $r=-0.12$, lo cual evidencia el avance de los créditos ante la caída del costo del dinero que generó resultados favorables en el producto bruto interno (PBI). El gasto público participó con un débil empuje a este indicador macroeconómico, hecho que se aprecia en la correlación del 0.014. Conclusión: Las medidas monetarias y fiscales, materializadas en el aumento del circulante, la dinamización de los créditos, disminución de las tasas de interés y aumento del gasto público evitaron una mayor profundización de la crisis económica, generando una leve pero sostenida recuperación del PBI y del empleo, pero manteniendo siempre la estabilidad macroeconómica.

Palabras clave: Producto bruto interno; tasa de interés; política monetaria; política fiscal; crisis sanitaria.

\begin{abstract}
Objective: Determine how the monetary and fiscal measures implemented under a global health crisis scenario mitigated the negative effects on the national economy. Method: Descriptive, correlational, cross-sectional, and non-experimental research. Other information, obtained from official government sources, was used to perform statistical measurements. Results: A negative Pearson correlation was found between the issuance of money and the interest rate $r=-0.46$, which explains the fall in interest rates due to increases in working capital. The same type of relationship was found between the interest rate and business loans, with the coefficient $r=-0.12$, which shows the advance of loans due to the fall in the cost of money that generated favorable results in gross national product (GNP). Public spending contributed with a weak boost to this macroeconomic indicator, a fact that can be seen in the correlation of 0.014 . Conclusion: The monetary and fiscal measures, materialized in the increase in working capital, the revitalization of loans, a decrease in interest rates and an increase in public spending, prevented a further deepening of the economic crisis, generating a slight but sustained recovery of GNP and employment, but always maintaining macroeconomic stability.
\end{abstract}

Keywords: Gross national product; interest rate; monetary policy; fiscal policy; health crisis. 


\section{INTRODUCCIÓN}

Todo el planeta está sufriendo el impacto de la crisis sanitaria, cuyos efectos perniciosos han puesto a prueba un tipo de paradigma económico, el cual se ha sustentado en el accionar de los mercados guiados, en cierto modo, por una mano invisible, tal como lo decía Smith (1997). En esta misma dirección de análisis, Pizarro (2020) enfatiza que "el virus ha dejado al desnudo la incapacidad del Estado y del modelo económico neoliberal para proteger a la sociedad, y a todos sus miembros" (p. 1035).

El paradigma económico, el cual también se conoce como globalización, si bien dio buenos resultados macroeconómicos, especialmente en aquellos países que pusieron en práctica la apertura de los mercados, al mismo tiempo, generó fisuras o asimetrías que profundizaron las diferencias entre las economías de los países e incluso en las estructuras de la sociedad. Sobre este punto, Kristjanpoller y Contreras (2017) toman como referencia a Sen (2001), quien sostiene que los efectos del fenómeno de la globalización no suceden de manera uniforme, todo depende de la región y del grupo que se elija, así como de los indicadores de la prosperidad económica, argumento que va dentro de la línea de análisis de las asimetrías económicas y sociales.

Dentro de este avance macroeconómico global, los beneficios del crecimiento no fueron suficientes para eliminar las asimetrías sociales, gestándose por ello una población invisible totalmente alejada de los beneficios que generaba la economía global y que es común en América latina y el Caribe, considerada la región más desigual del mundo (Cabezas, 2020). Para mitigar estos impactos negativos, los esfuerzos fiscales de los Gobiernos han llegado a materializarse a través de programas redistributivos y, en paralelo, flexibilizar la política monetaria, por ello, Rodríguez (2021) señala que “(..) el objetivo central de las políticas macroeconómicas - fiscal y monetaria - debería ser elevar el nivel de empleo, la fuente principal de ingresos de los hogares" (p. 252).

Asimismo, es importante centrar la atención en los multiplicadores fiscales y monetarios, como estipula la teoría económica, para así dinamizar la economía o en todo caso detener la caída vertiginosa del producto bruto interno (PBI), afirmación basada en la teorización macroeconómica de Dornbusch, Fischer y Startz (2010). Si bien los bancos centrales gozan de autonomía ante escenarios de crisis, no pueden dejar que la economía se mueva solo por el esfuerzo fiscal. En el caso nacional, los técnicos del Banco Central de Reserva del Perú (BCRP), Montoro, Pérez y Herrada (2020) señalan lo siguiente:

Desde el anuncio del estado de emergencia a nivel nacional para contener la expansión del
COVID-19, el Banco Central de Reserva del Perú (BCRP) ha tomado medidas monetarias y financieras orientadas a promover el correcto funcionamiento de los mercados. Dichas medidas se enfocaron en la reducción del costo de financiamiento, la provisión de liquidez al sistema financiero y la reducción de la volatilidad de tasas de interés de largo plazo y del tipo de cambio. Se calcula que al 24 de julio de 2020 las operaciones de inyección del BCRP totalizan un monto de S/ 50,8 mil millones (7 por ciento del PBI). (p. 11)

La posición tomada por el BCRP expresa claramente la flexibilización de la política monetaria, cuyo objetivo fue revertir los efectos nocivos de la crisis sanitaria con desembolsos de S/ 22,1 mil millones vía operaciones repo de valores, monedas y cartera, y S/ 26,7 mil millones con garantía del Gobierno nacional en el marco del programa Reactiva Perú, medidas que, como se entiende, estaban dirigidas a mover el engranaje de la economía. Pérez (2016) argumenta que "el Banco Central aparte de cumplir su objetivo del control de la inflación (...) debe velar por el crecimiento económico" (p. 58).

El BCRP apuntaba a relajar la tasa de interés; sobre este punto, es importante tomar en cuenta lo expresado por Lahura (2017):

Existe una asociación lineal entre la tasa de interés de política monetaria y las tasas de interés del sistema bancario (...), adiciona, se observa que la tasa de interés de política monetaria está más correlacionada con las tasas de interés activas de corto plazo (préstamos menores a 360 días) que con las tasas de interés activas de largo plazo (préstamos mayores a 360 días). (p. 5)

Bajo este contexto, es necesario destacar la importancia de la tasa de interés para dinamizar la economía, debido a su rol transmisor entre el sector monetario y real de la economía (Mies, Morandé y Tapia, 2003). De este modo, los créditos y, por ende, las inversiones, en función de su grado de sensibilidad, deben de responder a la caída de las tasas de interés y así lograr los efectos multiplicadores esperados.

Es relevante mencionar la importancia del apalancamiento que ejercen las instituciones financieras en la actividad productiva, específicamente para salir del entrampamiento económico, como el generado por la pandemia del COVID-19; hecho que contrasta con lo señalado por Pessoa, Braga y Morais (2019), quienes tomando los aportes de Schumpeter (1934) y, posteriormente, de McKinnon (1973) y Shaw (1973), llegan a demostrar la importancia 
de las instituciones financieras en el proceso de crecimiento económico, siendo las inversiones productivas el canal de transmisión para el logro de ese objetivo.

Empresas quebradas y operando por debajo de su potencial productivo, desempleo masificado, aumento de la extrema pobreza y el fortalecimiento de la informalidad son parte del escenario económico y social derivado de la pandemia sanitaria. A pesar de que se han ensayado políticas de rescate por el lado gubernamental, son mínimos los resultados alcanzados. Sobre el rol del Estado, Huerta (2020) explica lo siguiente:

La dimensión de la crisis que enfrentamos actualmente exige un Estado fuerte, que asuma un papel rector y que recupere el manejo soberano de la política monetaria, fiscal, financiera y comercial para contar con los recursos y hacer la asignación necesaria para superar la pandemia y la crisis económica. (p. 59)

Si bien es una posición ortodoxa de corte keynesiano, es necesario que la participación del Estado sea predominante para intentar revertir la caída de la economía vía ejecución de políticas públicas descentralizadas y de inclusión social generalizada, cuyo costo financiero se podría materializar en un mayor déficit fiscal o endeudamiento público, tal como lo considera el Fondo Monetario Internacional (2021).

Ante este escenario de elevado riesgo, estancamiento económico y sin signos de una pronta reactivación económica, los Gobiernos han apostado por laxar las políticas fiscales complementando con programas agresivos de tipo monetarios que han llevado a las tasas de interés muy cerca a cero. Para corroborar lo expresado, en el caso peruano, se presenta el programa Reactiva Perú, que según Sánchez y Castro (2020) "ha potenciado la transmisión de la reducción de la tasa de interés de referencia del Banco Central de Reserva al resto de tasas del sistema financiero" (p. 20). Sin embargo, es preciso señalar que existe la posibilidad de caer en la trampa de la liquidez explicada por Keynes (1965), ya que, a pesar de los esfuerzos, no se han visto mayores impactos en las inversiones productivas $y$, por ello, se percibe la neutralidad en el PBI.

La importancia de la política fiscal para impulsar el crecimiento se puede encontrar en la investigación realizada por Cerón (2013), quien afirma que "la evaluación de los efectos de las decisiones fiscales sobre la producción de un país constituye un input fundamental para las decisiones de política económica" (p. 178).

¿Cuál es el grado de relación que existe entre la gestión de las políticas monetarias y fiscales, y el comportamiento de la economía nacional, reflejado en el PBI, durante el periodo de crisis sanitaria del COVID-19?, interrogante que se desprende del análisis realizado y que orienta la presente investigación; además, permite formular las siguientes hipótesis: "La magnitud de la relación que existe entre la política monetaria y las tasas de interés es negativa y débil"; de igual forma, "la magnitud de la relación entre la tasa de interés y los créditos es negativa y muy débil"; mientras que "la magnitud de la relación entre los créditos privados anualizados y el PBI es positiva y muy débil". No obstante, también se estima para cada caso la magnitud de la determinación entre las variables antes mencionadas.

$\mathrm{Al}$ analizar el comportamiento de las variables monetarias y reales se llega a evidenciar el avance de la economía nacional bajo el escenario de crisis global.

\section{MATERIAL Y MÉTODOS}

La investigación fue de tipo descriptiva, correlacional, transversal y no experimental, cuyos resultados permitieron evidenciar el avance de la economía peruana bajo escenario de crisis sanitaria global. Para el desarrollo de la investigación, se utilizó información de la revista especializada que edita el BCRP denominada Nota Semanal, documento que contiene el comportamiento histórico de las variables monetarias y reales que fueron utilizadas en la investigación. Además, se hizo uso de las estadísticas económicas que procesa y publica trimestral y anualmente el Instituto Nacional de Estadística e Informática (INEI), así como de las publicaciones de la Superintendencia de Banca, Seguros y AFP (SBS) de donde se obtuvo información sobre los créditos empresariales. Se procesó y analizó información mensual para el periodo 2018-2020.

El circulante, los tipos de interés, créditos empresariales, gasto público, el PBI, y el empleo fueron las variables que se consideraron para realizar el estudio de investigación. Para establecer el grado y tipo de relación entre el circulante y la tasa de interés, y este indicador monetario con los créditos empresariales, se utilizó el coeficiente de correlación de Pearson (r), el cual resulta adecuado para medir el grado de asociación lineal que existe entre estas variables, y el coeficiente de determinación $\left(R^{2}\right)$. Estos estadísticos también fueron aplicados para contrastar el comportamiento de los créditos y del gasto público con el PBI, agregado macroeconómico que a su vez fue relacionado con la creación de empleo en el periodo de estudio.

\section{RESULTADOS}

Debido a la pandemia, la economía peruana ha experimentado una de las peores crisis. El avance macroeconómico obtenido en estos últimos años ha tenido un 
retroceso significativo, el cual es evidenciado con la caída del PBI que, según el BCRP (2021), llegó a -11.1\% en el año 2020 , fenómeno que además ha comprometido la sostenibilidad del mercado laboral y en donde se aprecia una tasa de desempleo del 13.0\%, según las estadísticas publicadas por el Instituto Nacional de Estadística e Informática (2021).

En esta misma dirección se encuentran las demás economías del mundo, cuya contracción económica ha conllevado a una caída del PBI global del 2.5\% (Fondo Monetario Internacional, 2021). Cabe señalar que solo la economía china experimentó un crecimiento del $1.7 \%$, tasa bastante lejana a la que siempre lograba y que ejercía efectos multiplicadores al resto de las economías del mundo, especialmente, en aquellos países que sustentan su economía con exportaciones de productos primarios.

El escenario de crisis de la economía peruana tenía que destrabarse o, en todo caso, amortiguar caídas más profundas debido al problema sanitario global. Por ello, la autoridad monetaria, juntamente con el Poder Ejecutivo (Ministerio de Economía y Finanzas), optaron por flexibilizar la política monetaria y fiscal teniendo en cuenta el límite máximo de la tasa de inflación, el cual, según el BCRP debe ser del 3\%, condición necesaria para mantener estable la economía.

En el año 2020, el BCRP flexibilizó la emisión de circulante y con ello la oferta monetaria, haciendo posible que disminuya la tasa monetaria, la cual se corrobora cuando se contrastan las cifras para ambas variables, evidenciando un coeficiente de correlación $\mathrm{r}=-0.44$ y determinación $\mathrm{R}^{2}=0.20$, tal como se aprecia en la Figura 1.
Dichas cifras señalan que esta tasa de interés experimentó un comportamiento opuesto a la emisión de circulante, buscando la mejora de la demanda agregada. El resultado se puede estabilizar aún más cuando se contrastan cifras de tales variables entre junio 2018 y diciembre 2020, donde se obtiene una correlación de $\mathrm{r}=-0.46 \mathrm{y}$, por ello, se puede afirmar que la aplicación de una política monetaria expansiva generó caídas en las tasas de interés. Por ende, al ser el mecanismo de transmisión, conllevó a mejores indicadores macroeconómicos para la economía peruana.

Si una autoridad monetaria practica políticas agresivas aumentando el circulante y con ello la oferta monetaria o liquidez, dependiendo cuanto sea la emisión y la temporalidad del impacto, las tasas de interés deberían caer; es decir, todas las tasas que se ponen en práctica en el mercado de corto plazo deben sensibilizarse con tendencia a la caída y, de esta forma, influir en variables reales como son la inversión privada.

El objetivo del BCRP era bajar la tasa de interés y, con ello, dinamizar la intermediación financiera vía canalización de nuevos créditos; sin embargo, debido a la información contrastada que va de junio 2018 a diciembre 2020, ante la caída de la tasa de interés interbancaria - cuya sensibilidad medida a través de la desviación típica llega al 0.6\%los créditos bancarios en moneda nacional reaccionaron positivamente, caso que se esperaba ante la medida del BCRP y que puede corroborarse con el coeficiente de correlación $\mathrm{r}=-0.12$.

Si bien la correlación entre ambos componentes monetarios es débil, debido a la reacción de los créditos, se evitó



Figura 1. Circulante y tasa interbancaria (TIB) (junio 2018-diciembre 2020)

Fuente: Elaboración propia sobre la base del Banco Central de Reserva del Perú (2021) 
que la economía siga profundizando los retrocesos en la actividad productiva. Se debe tener en cuenta que en el marco del Programa Reactiva Perú se podían otorgar garantías del Gobierno Nacional hasta por la suma de 60 mil millones de soles. Esta medida estaba orientada al sector empresarial, en especial hacia las mype, buscando evitar mayores contracciones económicas y el quiebre en la cadena de pagos que, en cierta forma, trastocaría la estabilidad financiera. Así, al contrastar las cifras de la tasa activa promedio y los créditos a las empresas privadas, se observa, a través del coeficiente de correlación $(\mathrm{r})$ y determinación $\left(\mathrm{R}^{2}\right)$, que las medidas monetarias no tuvieron la contundencia para reactivar la economía en época del COVID-19.

La incidencia de la tasa de interés en los créditos, la cual según los resultados estadísticos es débil e inverso, se complementa cuando se contrastan los créditos privados y el PBI. La flexibilización de la política monetaria, a través del mecanismo de transmisión, que es la tasa de interés, debe tender a dinamizar el PBI vía el cambio de las inversiones y el consumo, que son componentes de la demanda agregada.

La sensibilidad de las inversiones a la tasa de interés es sumamente importante, ya que el grado de cambio de estos flujos repercute en la actividad productiva; no obstante, en época de crisis sanitaria, en donde los escenarios son inciertos y los riesgos por invertir son altos, las medidas monetarias en ciertos casos dejan de tener los efectos esperados, llegando a formarse una especie de trampa de liquidez. Pero, en el caso peruano, el esfuerzo monetario logró resultados positivos en el comportamiento del PBI, y si bien no se lograron cifras de crecimiento sostenido en el periodo de crisis global, su amortiguamiento fue importante para evitar la profundización de la crisis hasta lograr una cifra marginal, pero positiva del PBI en el mes de diciembre.

En la Figura 2, se aprecia el comportamiento del PBI del mes de enero a diciembre de 2020, en donde se observa la lenta recuperación del PBI, debido a la flexibilización de la política fiscal y monetaria.

$\mathrm{Al}$ contrastar las cifras para el periodo enero a diciembre 2020 , se obtiene una correlación débil $(r=0.16)$ que, si bien el grado de relación entre estas dos variables no tienen mayor fuerza, sí indica la incidencia de los créditos privados en el comportamiento del PBI.

Dicho esto, su comportamiento en los periodos de crisis se fue corrigiendo paulatinamente. La cifra positiva de correlación indica que, a medida que la tasa de crecimiento de los créditos disminuía al transcurrir la pandemia, el PBI iba desacelerando su decrecimiento, resultado obtenido además por las medidas fiscales implementadas por el Poder Ejecutivo.

La Figura 3 muestra el recorrido de los créditos anualizados y el PBI, en donde se aprecia la forma en la cual los intermediarios financieros apalancan el proceso de recuperación económica, que si bien no son cifras positivas, sí generan un efecto de amortiguamiento para contener la caída de este indicador macroeconómico que en el mes de abril alcanzó un piso del $-39.10 \%$, pero que en los meses siguientes mostró una continua recuperación de las colocaciones crediticias por parte de las entidades financieras. Particularmente, en abril, avanzó a una cifra en promedio

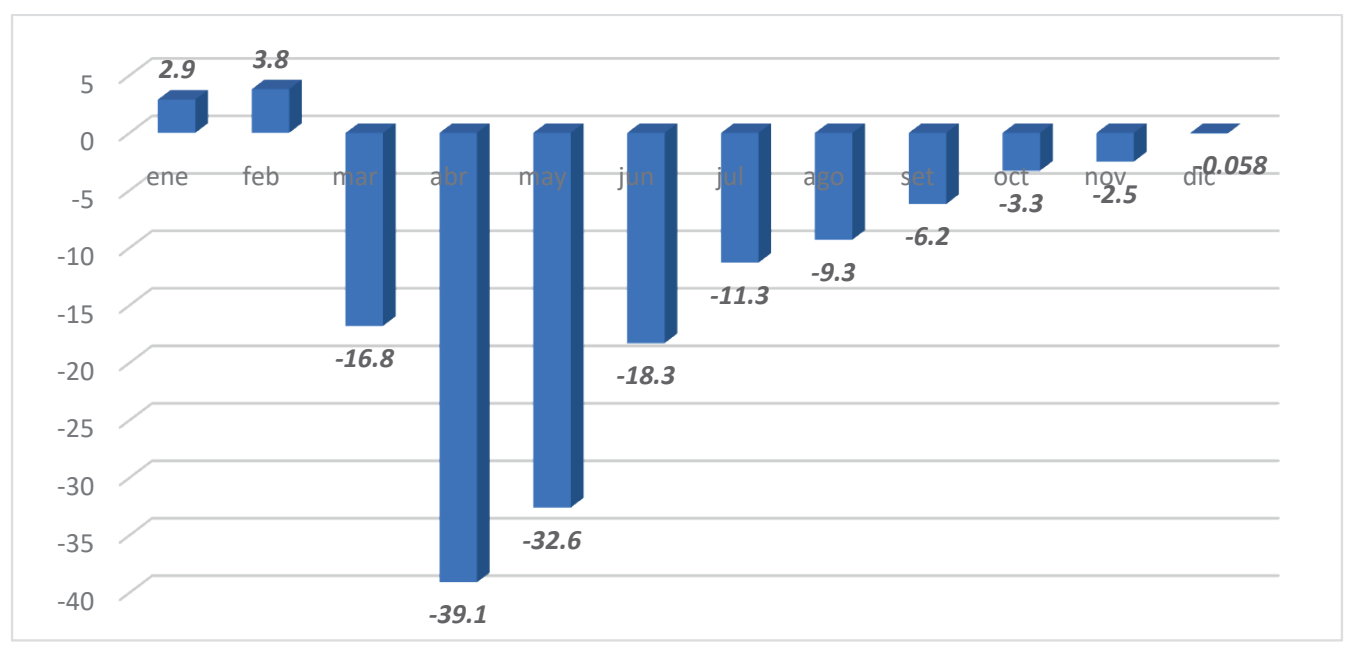

Figura 2. Perú y PBI (enero 2020-diciembre 2020) (Var. \%)

Fuente: Elaboración propia sobre la base del Banco Central de Reserva del Perú (2021) 


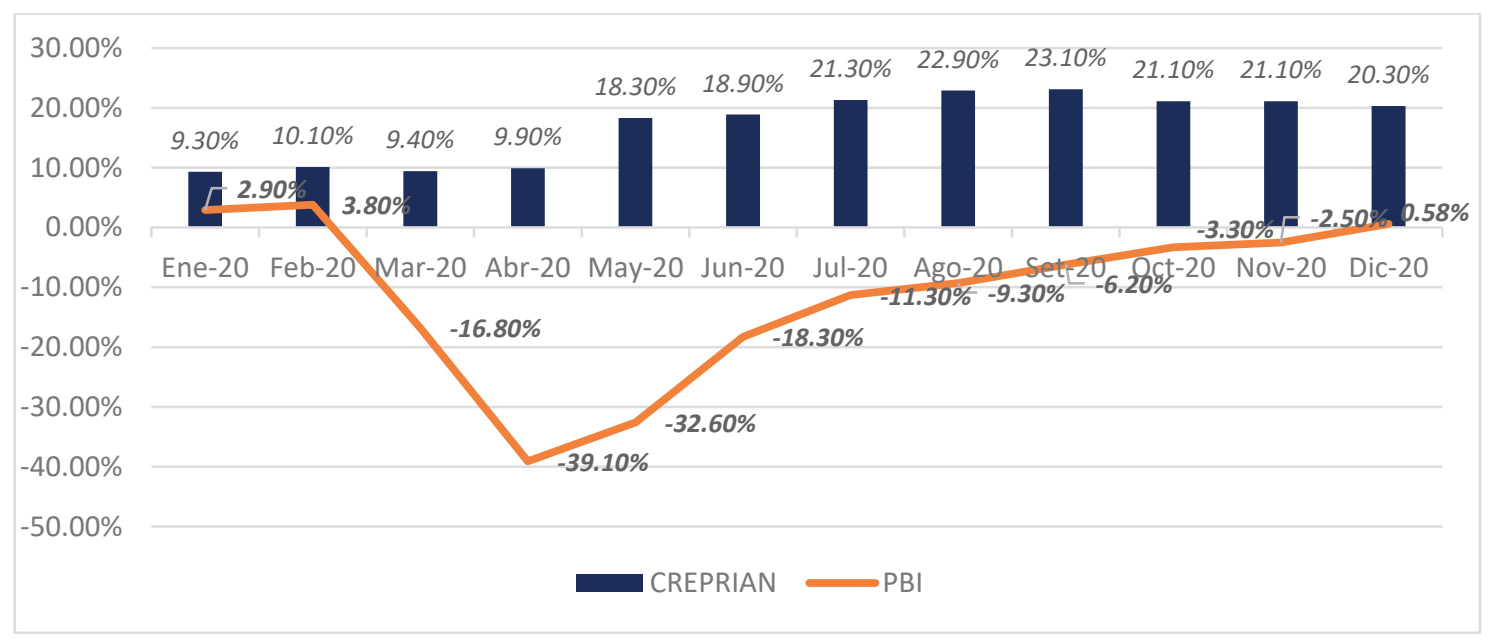

Figura 3. Crédito privado anual y PBI (enero 2020-diciembre 2020) (Var. \%)

Fuente: Elaboración propia sobre la base del Banco Central de Reserva del Perú (2021)

del $20 \%$ apoyado en tasas de interés que motivaban a la búsqueda de mayores apalancamientos financieros gracias a las decisiones de política monetaria del BCRP.

El empuje de la economía no solo estuvo por el lado monetario, sino también por el fiscal, mediante programas de subsidios (especialmente a los de mayor vulnerabilidad económica), los cuales fueron desembolsados en tramos y que incluso forzaron a un endeudamiento externo por 100 años, monto que bien podría comprometer el equilibrio fiscal, debido a que estos fondos están destinados a financiar gastos corrientes.

Incurrir en gastos públicos es dinamizar la demanda agregada y con ello el PBI, pero la correlación encontrada solo muestra la magnitud del coeficiente de correlación de $r=0.014$ que estaría indicando la débil incidencia del esfuerzo presupuestal para contener la caída del PBI o, en todo caso, para generar escenarios de crecimiento.

Sin embargo, se debe tener presente que este esfuerzo presupuestario estaba básicamente dirigido a financiar gastos corrientes que, según la literatura macroeconómica, debe generar efectos multiplicadores (Dornbush, Fischer y Startz, 2015).

Si bien las medidas monetarias y fiscales actuaron como mecanismos de contención de los efectos perniciosos de la crisis sanitaria, estas no fueron suficientes para evitar el debilitamiento del mercado laboral, en donde se experimentó el aumento del desempleo que, según el Instituto Nacional de Estadística e Informática (2020), al final de 2020 llegó al 13.0\%, complementado con la menor creación de puestos de trabajo por parte del sector privado.
Tal como se puede apreciar en la Figura 4, de enero a diciembre de 2020, en el sector privado, se perdieron 134 mil puestos de trabajo, que hubieran sido más de no haber recurrido a los instrumentos monetarios y fiscales que macroeconómicamente apalancaron la demanda agregada.

Los efectos nocivos de la crisis sanitaria en el mercado laboral se pueden apreciar en el comportamiento del empleo en forma anualizada con resultados negativos en casi todo el período 2020, excepto en los meses de enero y febrero, fechas en donde aún no se profundizaba la crisis sanitaria que logró avanzar en un $3,1 \%$ y 2,8\%, respectivamente. Al contrastar las cifras del empleo con el PBI, se puede observar, además, cómo las tasas negativas comienzan a desacelerarse, es decir, como producto de una menor caída de la actividad económica, también lo hace la tasa de desempleo, cuyo recorrido, aún en el cuadrante negativo, comienza a ser más moderado.

Por otro lado, en un informe de la Superintendencia de Banca, Seguros y AFP (2020), se menciona lo siguiente:

A diciembre 2020 se observa una reducción de las tasas de interés promedio en todos los segmentos. La mayor caída se da en los créditos a microempresas, lo que estaría explicado por las menores tasas de los créditos otorgados en el marco de los programas Reactiva Perú y Fondo de Apoyo Empresarial a la MYPE, programas de Gobierno que buscan garantizar el financiamiento para capital de trabajo de las empresas y personas naturales con negocio ante el impacto económico de la pandemia. (p. 7) 


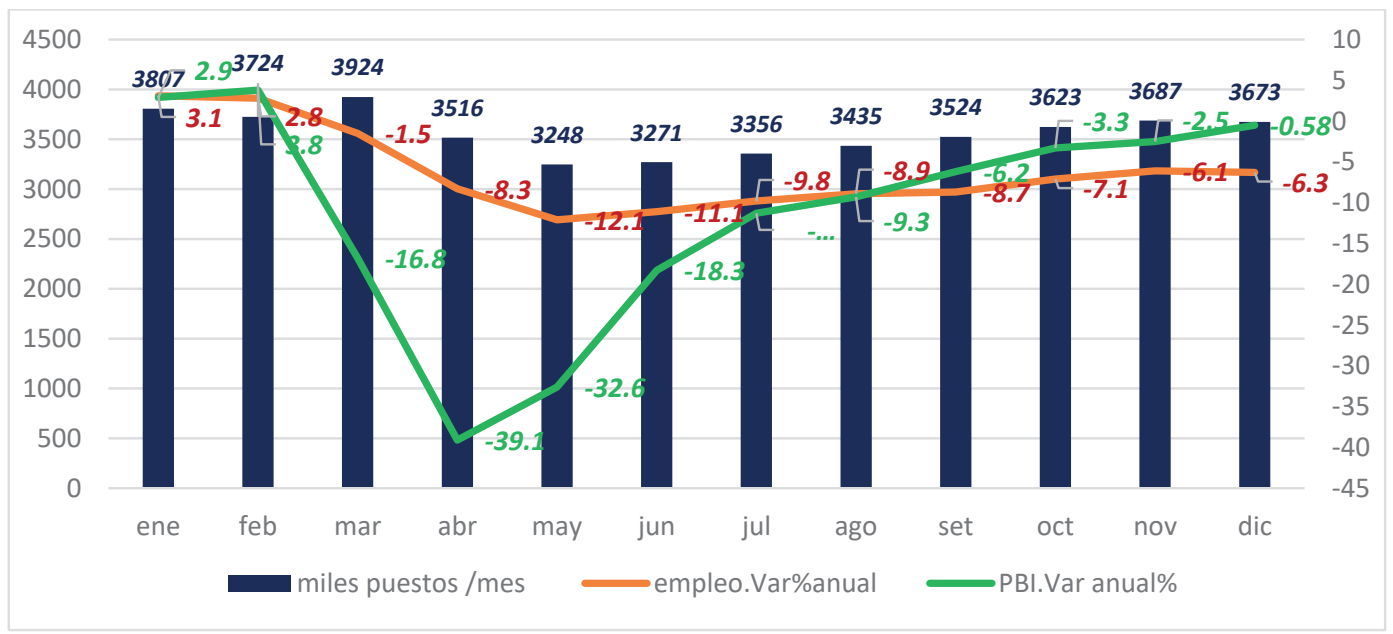

Figura 4. Creación de trabajo privado y el PBI (enero-diciembre 2020)

Fuente: Elaboración propia sobre la base del Banco Central de Reserva del Perú (2021) y el Instituto Nacional de Estadística e Informática (2021)

A la luz de las cifras del informe, se puede apreciar que aquel sector que aglomera el 95\% de las empresas en el Perú, en plena pandemia y con una alta crisis económica, no logró obtener los beneficios de apalancamiento financiero debido a que la elevada tasa de interés que, a diciembre de 2020, llegaba al 40.1\% y que, para los créditos microempresariales, limitaba el aporte de este sector en proceso de recuperación económica.

A pesar de la creación del Programa Reactiva y de la flexibilización de las medidas monetarias, el costo de los créditos seguía castigando a dicha actividad económica que, por naturaleza, es generadora de empleo. La política monetaria no fue suficiente para lograr reducir esa asimetría de tasas de interés, como se ve en la Figura 5, donde las empresas corporativas que, aparte de concentrar los fondos crediticios, asumen menores tasas de interés, situación que es característica en estas organizaciones con o sin crisis sanitaria.

El programa monetario apalancó a las grandes empresas conocidas como Principales Contribuyentes (PRICOS), que son las que más aportan tributariamente al Estado, lo cual se aprecia con las bajas tasas de interés y los montos que se destinan; aun así, la fluidez de los créditos a las micro y pequeñas empresas tuvo limitaciones para potenciar sus actividades productivas y el empleo.

Ciertamente, la política monetaria puede flexibilizar la tasa monetaria y, con ello, potenciar las inversiones e impulsar la demanda agregada, pero los costos efectivos de los créditos son colocados por las instituciones financieras en función de los riesgos que asumen. Tomando en cuenta lo señalado por el Ministerio de Economía y Finanzas (2020), el Programa de Reactivación — sustentado por las medidas monetarias - estaba enfocado en dar continuidad a la cadena de pagos, otorgando créditos a las micro, pequeñas, medianas y grandes empresas y, de esta forma, evitar además que se generen cuellos de botellas en los cumplimientos financieros, pilares importantes para generar sostenibilidad a la actividad económica.

En la Tabla 1, se puede observar que las cifras proyectan claramente una concentración de los créditos a favor de los corporativos y grandes empresas, alcanzando en el 2020 el $34 \%$, mientras que para las micro y pequeñas empresas fue solamente de $14 \%$, mostrando las profundas asimetrías en la diversificación de los créditos que no pudieron ser corregidas con el Programa Reactiva Perú. Sin embargo, este Programa pudo fortalecer el circuito financiero en época de crisis sanitaria, a pesar de las debilidades del Sistema Financiero en Perú, actuando como mecanismo de contención para evitar que se profundice la crisis económica.

Es importante mencionar que, si el Gobierno no hubiera materializado este tipo de apalancamiento, las colocaciones crediticias hubieran caído en un $4.8 \%$, montos intermediados que hubieran complicado dar soporte a los embates de la crisis sanitaria y, por ende, a la económica.

\section{DISCUSIÓN}

La crisis sanitaria ha generado severos problemas económicos a nivel mundial, originando caídas sostenidas del PBI que además comprometieron la sostenibilidad del mercado financiero y laboral. El estancamiento económico en todos 


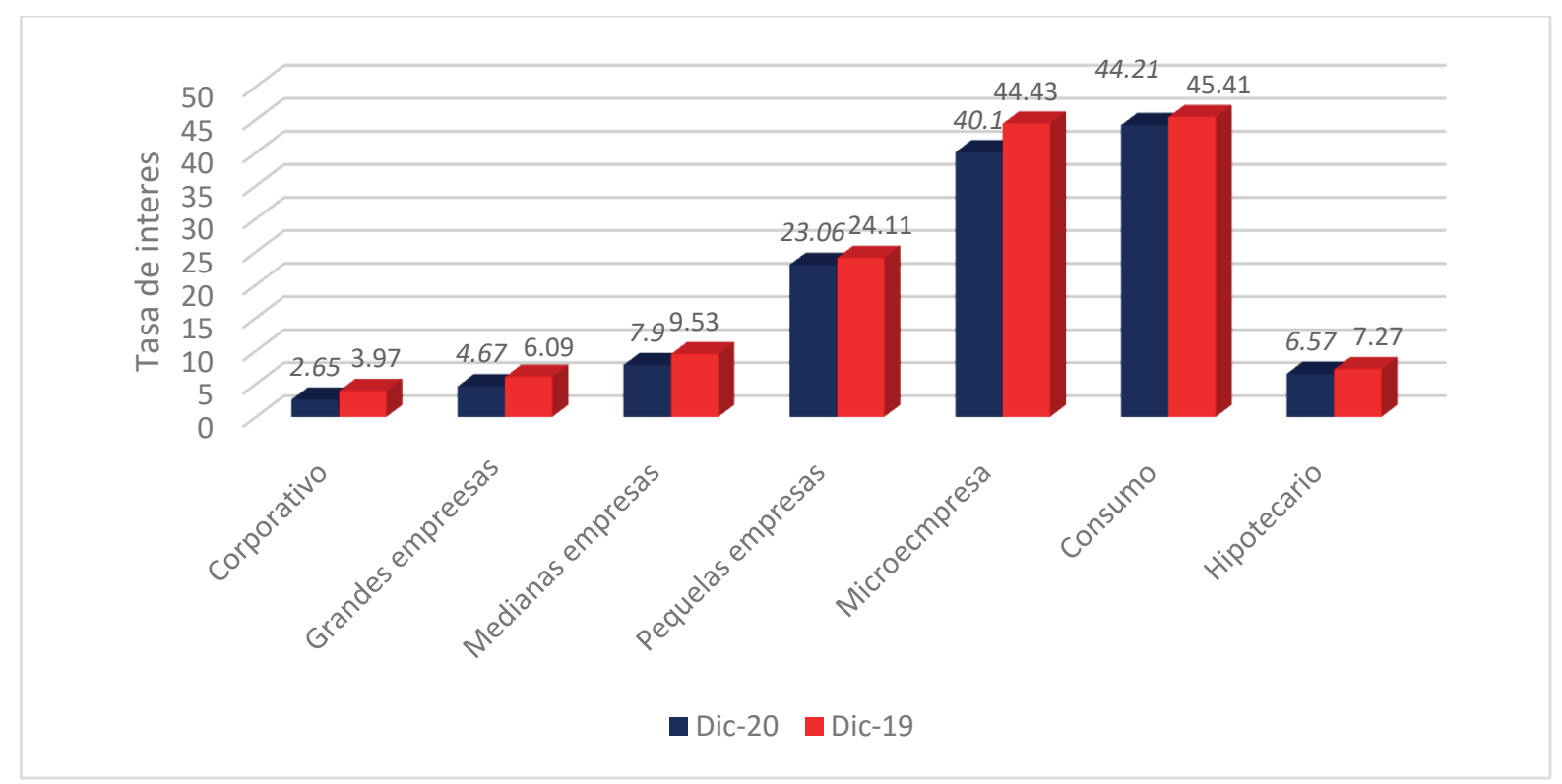

Figura 5. Tasa de interés promedio del sistema financiero por sectores económicos (diciembre 2019 y diciembre 2020)

Fuente: Elaboración propia sobre la base de la Superintendencia de Banca, Seguros y AFP (2020)

Tabla 1

Sistema Financiero: composición de los créditos (diciembre 2020)

\begin{tabular}{|c|c|c|c|c|c|}
\hline & $\begin{array}{l}\text { Saldo Millones } \\
\text { S/ }\end{array}$ & $\%$ & $\begin{array}{l}\text { Var. \% } \\
\text { Anual }\end{array}$ & $\begin{array}{c}\text { Saldo } \\
\text { Millones S/ sin } \\
\text { COVID-19 }\end{array}$ & Var. \% Anual \\
\hline Corporativos y grandes empresas & 127760 & 0.34 & 7.4 & 110658 & $-7 \%$ \\
\hline Medianas empresas & 69950 & 0.19 & 53.6 & 44292 & $-2 \%$ \\
\hline mype & 54134 & 0.14 & 27.4 & 39350 & $-7.40 \%$ \\
\hline Consumo & 71421 & 0.19 & -7.1 & 71421 & $-7.10 \%$ \\
\hline Hipotecario & 53635 & 0.14 & 4.3 & 53635 & $4.30 \%$ \\
\hline TOTAL & 376900 & 1 & & 319356 & $-4.8 \%$ \\
\hline
\end{tabular}

Fuente: Elaboración propia sobre la base de la Superintendencia de Banca, Seguros y AFP (2020)

los sectores, con pocas opciones de ser revertido en un corto plazo, fue la característica del problema sanitario global del COVID-19. Con ello, se paralizaron o debilitaron todas las industrias con un severo castigo al mercado laboral que mermó la capacidad de compra de los consumidores. Por esta razón, el Gobierno, por medio del BCRP, optó por realizar operaciones de rescate, ejecutando políticas fiscales y monetarias expansionistas, tal como se evidencia en el análisis realizado por Montoro, Pérez, Herrada (2020). La caída de las tasas de interés, tanto la interbancaria y la monetaria, que tenían como fin agilizar los créditos empresariales, marcó la pauta de las demás tasas de interés. Si bien la incidencia no fue tan contundente, como se puede apreciar con el coeficiente de correlación, la agilización de los créditos permitió amortiguar la caída del PBI; dicho de otra manera, estas operaciones de apalancamiento financiero evitaron profundizar las crisis económicas.

Por ello, ante escenarios de crisis y la pasividad de los capitales privados, es necesaria la intervención del Estado, a través de ejecuciones de gasto, tal como se evidencia en las cifras presentadas que, al contrastar con el PBI, se observa que actuó como factor amortiguador de los efectos perniciosos de la crisis sanitaria. Afirmación que contrasta con lo señalado por Cerón (2013), quien explica sobre las decisiones fiscales para generar impulsos en la producción, 
más aún en periodos de recesión económica. La política monetaria y fiscal tuvo resultados, pero la contundencia de estos no fue la esperada, ya que no se logró contener los desempleos masivos; sin embargo, sí alcanzó a generar espacios económicos para fundamentar crecimientos futuros. La tasa de interés, actuando como mecanismo de transmisión y debido a su sensibilidad ante la política monetaria, empujó el sector real de la economía, pero con serias limitaciones para alcanzar cifras de crecimiento, tal como es característico cuando se presentan incertidumbres y riesgos en la economía, línea de análisis que encaja con lo explicado por Mies, Morandé y Tapia (2003), quienes sustentan la importancia de la tasa de interés en el rol dinamizador de la economía que además se complementa con la de Sánchez y Castro (2020), quienes basados en el Programa Reactiva Perú fundamentan las bondades de la tasa de interés para lograr objetivos de crecimiento.

Se concluye que el aumento del circulante, una reducción de las tasas de interés y los desembolsos de créditos empresariales, enmarcados dentro de la política monetaria expansiva, complementados con el empuje fiscal vía gasto público, redujeron los efectos negativos de la crisis sanitaria, hecho evidenciado en la lenta, pero sostenida recuperación del PBI y el empleo que mantuvo siempre la estabilidad macroeconómica.

Sobre la base de lo señalado, surgen interrogantes que pueden dar apertura a nuevas investigaciones que generen aportes para dar mayor robustez al desempeño de la economía peruana: ¿Cómo el presupuesto público, como herramienta de política fiscal, podría ser utilizado para minimizar los espacios de vulnerabilidad en la sociedad? y ¿ por qué una eficaz política monetaria, a través de la tasa de interés, podría acelerar el crecimiento económico, pero con estabilidad?

\section{REFERENCIAS}

Banco Central de Reserva del Perú. (28 de enero de 2021). Nota Semanal $N^{\circ} 4$. Recuperado de https://www.bcrp. gob.pe/publicaciones/nota-semanal.html

Cabezas, C. (2020). Pandemia de la COVID-19: Tormentas y retos. Revista Peruana de Medicina Experimental y Salud Pública, 37(4), 603-604. Recuperado de http://www.scielo.org.pe/scielo, php?pid=S1726-46342020000400603\&script=sci arttext

Cerón, J. A. (2013). Los multiplicadores fiscales: Una revisión de la literatura empírica. Revista de Economía Mundial, (34), 175-209. Recuperado de https://www. redalyc.org/articulo.oa?id $=86628239008$
Dornbusch, R., Fischer, S., \& Startz, R. (2015). Macroeconomía (12. ${ }^{a}$ ed.). México D.F.: McGraw Hill.

Fondo Monetario Internacional. (Octubre de 2021). La recuperación en tiempos de pandemia. Cuadro de proyecciones. Recuperado de https://www.imf.org/es/ Publications/WEO/Issues/2021/10/12/world-economic-outlook-october-2021

Instituto Nacional de Estadística e Informática. (2021). Situación del Mercado Laboral en Lima Metropolitana. Recuperado de https://www.inei.gob.pe/media/MenuRecursivo/boletines/03-informe-tecnico-mercado-laboral-dic2020-ene-feb2021.pdf

Huerta, A. (2020). Propuestas para enfrentar la crisis económica producida por la pandemia 2020. Revista de la Academia de México de la Ciencia, 71(3), 58-65. Recuperado de https://www.revistaciencia.amc.edu.mx/ index.php/vol-71-numero-3-e

Keynes, J. M. (1965). Teoría general de la ocupación, del interés y el dinero. México D.F.: Fondo de Cultura Económica.

Kristjanpoller, W., \& Contreras, R. (2017). Globalización y desigualdad: Evidencia en América Latina. Lecturas de Economía, (87), 9-33. https://doi.org/10.17533/udea. $\underline{\text { le.n87a01 }}$

Lahura, E. (2017). El efecto traspaso de la tasa de interés de política monetaria: 2010-2017. Revista Moneda, (170), 4-7. Recuperado de https://www.bcrp.gob.pe/ publicaciones/revista-moneda/revista-moneda-170. $\underline{h t m l}$

Mies, V., Morandé, F., \& Tapia M. (2003). Política monetaria y mecanismo de transmisión monetaria. Recuperado de https://www.cemla.org/PDF/premiobc/pub-libpremio2003-B.pdf

Montoro, C., Pérez, F., \& Herrada, R. (2020). Medidas del BCR frente a la pandemia del nuevo coronavirus. Revista Moneda, (182), 10-18. Recuperado de https:// www.bcrp.gob.pe/publicaciones/revista-moneda/revista-moneda-182.html

Pérez, J. (2016). Política monetaria y su impacto en el crecimiento económico de México de 1995 a 2015 (Tesis de maestría, El Colegio de la Frontera Norte, México.) Recuperada de https://colef.repositorioinstitucional. $\underline{\mathrm{mx} / \mathrm{jspui} / \mathrm{handle} / 1014 / 474}$

Pessoa, F., Braga, M., \& Morais, G. (2019). Crecimiento económico y desarrollo financiero en el Brasil: Investigación a partir del modelo de regresión flexible. Re- 
vista CEPAL, (128), 101-123. Recuperado de https:// repositorio.cepal.org/handle/11362/44739

Pizarro, R. (2020). Un paso más allá de la Covid-19. El Trimestre Económico, 87(348), 1033-1057. https://doi. org/10.20430/ete.v87i348.1174

Rodríguez, J. A. (2021) Situación económica, política fiscal y pandemia en Colombia. Revista de Economía Institucional, 23(44), 249-263. Recuperado de http:// www.scielo.org.co/scielo.php?script=sci arttext\&pi$\underline{\mathrm{d}=\text { S0124-59962021000100249 }}$

Sánchez, E., \& Castro F. (2020). Reactiva Perú y la estabilidad del Sistema Financiero. Revista Moneda, (182), 19-23. Recuperado de https://www.bcrp.gob.pe/publicaciones/revista-moneda/revista-moneda-182.html
Smith, A. (1997). La teoría de los sentimientos morales. Madrid: Estudio Alianza Editorial.

Superintendencia de Banca, Seguros y AFP. (2020). Evolución del sistema financiero. Diciembre 2020. Recuperado de https://www.sbs.gob.pe/estadisticas-y-publicaciones/estadisticas-/sistema-financiero 